\title{
Dentato-olivary dysplasia in sibs: an autosomal recessive disorder?
}

\author{
Tim Martland, Brian N Harding, Richard E Morton, Ian Young
}

\begin{abstract}
The cases of a brother and sister with dentato-olivary dysplasia are described. Both had severe developmental delay, severe epilepsy of early onset, evolving hypertonic quadriplegia, and death in early childhood. Postmortem examination of the brain in one child showed a particular form of dentato-olivary dysplasia. These children show many features in common with previously described cases of this condition, but this is the first report of occurrence in sibs.

(F Med Genet 1997;34:1021-1023)
\end{abstract}

Keywords: intractable epilepsy; severe developmental delay; cerebral malformation; dentato-olivary dysplasia

A small number of children with severe developmental delay, intractable seizures starting in the first months of life, an evolving spastic quadriplegia, and death in early childhood have been described in association with the pathological finding of dentato-olivary dysplasia at necropsy. ${ }^{1-3}$ We report two affected sibs with this condition. Previously reported cases have been isolated within families; our cases suggest this is an autosomal recessively inherited disorder.

\section{Department of}

Paediatric Neurology,

University Hospital,

Nottingham, UK

T Martland

Department of Histopathology, Great Ormond Street Hospital for Children, London, UK B N Harding

\section{Department of} Paediatrics, Derby Children's Hospital, Derby, UK

R E Morton

Department of Clinical Genetics, City Hospital, Nottingham, UK

I Young

Correspondence to: Dr Martland, Department of Paediatric Neurology, Ryegate Children's Centre Tapton Crescent Road, Sheffield S10 5DD, UK. whole body myoclonic jerks were noted at that time. His development then had not progressed beyond that of a newborn with no smiling, fixing, or following. Head circumference was on the 10 th centile. An initial electroencephalogram (EEG) was normal for age. Neurometabolic investigations (full blood count, routine biochemistry, thyroid function tests, chromosomes including fragile $\mathrm{X}$, blood and urine amino acids, urine organic acids, white cell enzymes, serum biotinidase, serum copper, congenital infection screen, blood uric acid, blood ammonia, cerebrospinal fluid protein, and glycine) were all normal. A repeat EEG at 3 months of age showed an abnormal record with very high amplitude activity. Frequent spike complexes were seen occurring independently on either side but more so on the left. The background activity was generally slowed. The record was not hypsarrhythmic. A computed tomography (CT) scan of the brain showed mild generalised cerebral atrophy only. Electroretinography (ERG) showed normal responses but visual evoked potentials (VEP) produced no response from either eye. Brain stem auditory evoked responses (BAER) showed increased $\mathrm{V}$ wave latencies bilaterally. The seizures failed to respond to phenobarbitone or pyridoxine but ACTH therapy produced a sustained improvement. He required a short and uneventful period of ventilation during an episode of viral bronchiolitis.

Examination at 6 months of age showed a passive child with little spontaneous movement and almost no vocalisation. There was increased tone in all four limbs with brisk tendon jerks. Head circumference remained on the 10th centile (and continued to grow appropriately until his death). He had just begun to smile, fix, and follow but had made no further developmental progress and later lost these skills. He was still having 20 to 30 seizures a day; by now these were mostly tonic seizures with occasional clonic movements.

He had no gastro-oesphageal reflux but had frequent respiratory infections probably related to episodes of aspiration. He died from respiratory disease at the age of 16 months. Necropsy of the brain showed generalised cerebral atrophy with widespread gliosis and myelin loss. Both the dentate and olivary nuclei were dysplastic. The dentate nucleus had the appearance of an oval plate, lacking a hilum, and was composed of interconnected islands of grey matter interspersed with myelin. The inferior olivary nuclei were unconvoluted, coarse, and hook shaped (figs 1 and 2). Other cerebellar and brain stem structures were normal.
CHIID 2

Child 2, a female, was born a year after her brother's death, at term, following a normal pregnancy and weighed $3200 \mathrm{~g}$. She appeared normal at birth but at 6 weeks of age developed symmetrical myoclonic jerks with neck flexion and limb extension. She showed delay in head control and, as with her brother, was late to smile and later lost this skill having made no other developmental progress. She developed severe rigidity of "lead pipe" type in all four limbs with marked dystonic posturing which later led to her developing a scoliosis. Head growth was maintained on the 10th centile for age. She was also a passive child with a paucity of spontaneous movement and made little spontaneous noise. An initial EEG was normal and her seizures showed a partial, but 


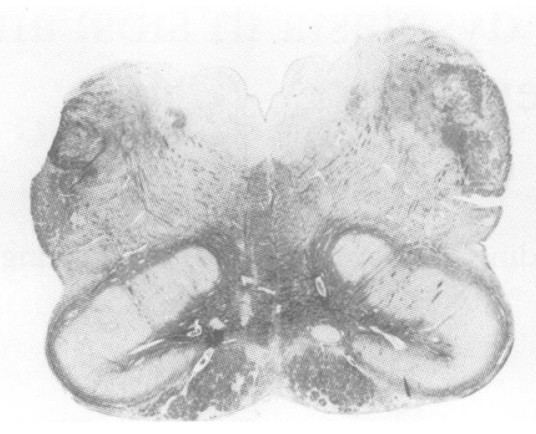

Figure 1 The inferior olives of sib 1 showing replacement of the normal, thin, convoluted cell band by a coarse, hook shaped structure.

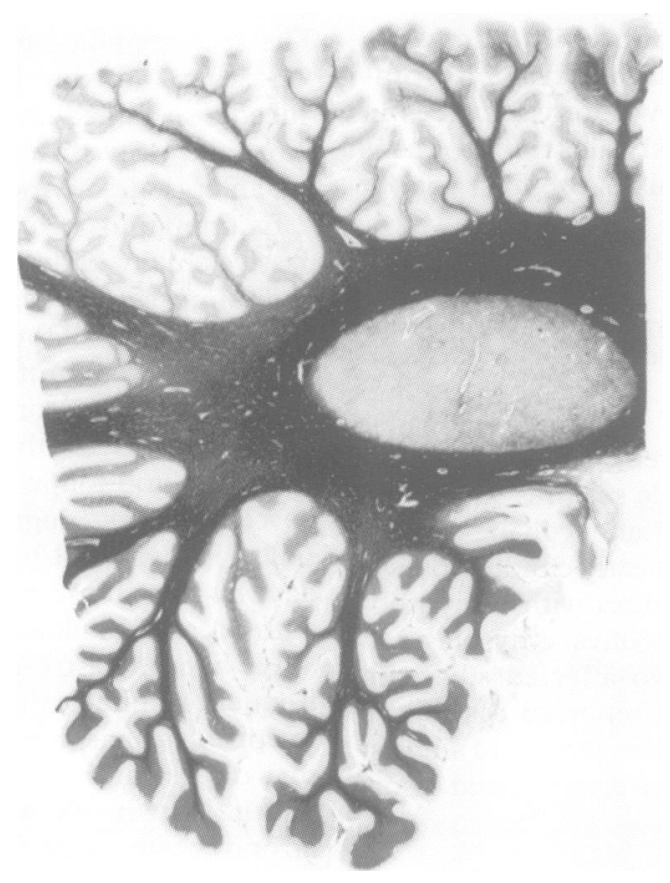

Figure 2 Dentate nucleus from sib 1 showing replacement of the thin, convoluted band of grey matter and white matter hilum by a solid ovoid structure.

sustained, response to clonazepam and valproate. Investigations, including routine haematology, biochemistry, urine and blood amino acids, urine organic acids, urine metabolic screen, chromosome analysis, very long chain fatty acids, and blood lactate, were normal. An MRI brain scan at 9 months of age showed generalised cerebral atrophy. A repeat EEG with simultaneous video recording showed high amplitude diffuse slow activity $(2-5 \mathrm{~Hz})$ and frequent independent spike discharges bilaterally. A seizure was associated with abrupt flattening of activity. Her seizures evolved into tonic seizures with some clonic movements during the first year of life, as had her brother's. ERG and VEP, performed earlier in life than in her brother, showed normal responses from both eyes. Later she showed no clinical evidence of visual function but these were not repeated. BAERs gave raised thresholds bilaterally with prolonged $V$ wave latencies. She also suffered from recurrent respiratory infections and died at the age of 2 years 4 months. A request for necropsy was refused.

\section{Discussion}

Although a considerable variety of anatomical forms of dysplasia may affect the dentate and olivary nuclei, ${ }^{4}$ the particular conformation described in our case 1 has only rarely been reported associated with severe developmental delay, intractable seizures starting in the first months of life, an evolving spastic quadriplegia, and death in early childhood. ${ }^{1-3}$ Indeed the clinical course of both sibs was similar to those previously described. The characteristic pathological findings at necropsy are of a dentatoolivary dysplasia (DOD) in which the inferior olives are hook shaped, coarse, and lacking undulations. The dentate nuclei show replacement of the thin, convoluted band of grey matter and white matter hilum by a solid ovoid structure. ${ }^{2}$ The pattern of abnormality is distinct from other olivary and dentate nuclei malformations, such as those seen in association with trisomy $18,{ }^{5}{ }^{6}$ Zellweger syndrome, ${ }^{7}$ and Joubert syndrome. ${ }^{8}$ The first child had findings typical of this condition on neuropathological examination of his brain.

The second child did not have a postmortem examination of her brain but there are striking clinical similarities between the children. The seizure types and EEG appearances are identical, both had neuroimaging showing generalised cerebral atrophy, both lost the early developmental milestones they had belatedly gained, and had negative neurometabolic investigations. There was evidence of brain stem dysfunction with prolonged $\mathrm{V}$ latency on BAER in both children. In addition there were marked similarities in the children's behaviour with passivity and paucity of movements. Neurological examination was almost identical in both. It seems extremely likely that the sister also had dentato-olivary dysplasia. MRI scanning of the brain in this second sib did not show any specific abnormalities.

The type of seizures the children had were clinically similar but not typical of infantile spasms or of the tonic seizures seen in early infantile epileptic encephalopathy (EIEE) (Ohtahara's syndrome), ${ }^{9}$ or of the erratic myoclonic seizures in early onset myoclonic epilepsy (EME). ${ }^{10}$ The association of a severe form of early onset epilepsy with a continually abnormal EEG and a poor prognosis would suggest that there is some overlap between these sibs and children with EIEE or EME. Brain malformation syndromes are a well recognised cause of EIEE.

It has been suggested that a "burstsuppression" EEG pattern is associated with DOD. Neither of our children had such an EEG appearance although both children had similar, very abnormal records. A burstsuppression pattern is not specific to dentatoolivary dysplasia, nor do all cases show this pattern (S Boyd, personal communication).

Previous reports of this condition have been isolated cases within families. This is the first report of the disorder occurring in two sibs and would suggest that this is an autosomal 
recessive condition. The parents are not related nor are there any neurological conditions in the extended family. There are autosomal recessive conditions associated with degeneration of brain stem nuclei, such as olivopontocerebellar atrophy and pontoneocerebellar hypoplasia. ${ }^{11}{ }^{12}$ These show a similar progressive course in older people. The dentate and inferior olive have a common embryological origin, being formed from neuroblasts migrating from the rhombic lip. This and their similar internal structure may explain why they are involved together in this disorder.

\section{Conclusions}

Two sibs are reported with dentato-olivary dysplasia suggesting an autosomal recessive inheritance for this rare condition. These cases also show the value of detailed neuropathological examination of children with a similar clinical presentation.

1 Harding B, Boyd SG. Early epileptic encephalopathy with suppression burst and olivary-dentate dysplasia. Neuropaediatrics 1992;23:336.
2 Harding BN, Boyd SG. Intractable seizures from infancy can be associated with dentato-olivary dysplasia. 7 Neurol Sci 1991;104:157-65.

3 Robain O, Dulac O. Early epileptic encephalopathy with suppression bursts and olivary-dentate dysplasia. Neuropaediatrics 1992;23:162-4.

4 Harding BN, Copp AJ. Malformations. In: Graham D, Lantos P, eds. Greenfield's neuropathology. 6th ed. London: Edward Arnold, 1996:397-533.

5 Michaelson PG, Gilles FH. Central nervous system abnormalities in trisomy 18. F Neurol Sci 1972;15:193-208.

6 Sumi SM. Brain malformations in the trisomy 18 syndrome. Brain 1970;93:821-30.

7 Evrard P, Caviness JR, Prats-Vinas J, Lyon G. The mechanism of arrest of neuronal migration in the Zellweger malformation; an hypothesis based upon cytoarchitectonic analysis. Acta Neuropathol 1978;41:109-17.

8 Freide RL, Boltshauser E. Uncommon syndromes of cerebellar vermis aplasia. 1 . Joubert syndrome. Dev Med Child Neurol 1978;20:758-63.

9 Ohtahara S, Ohtsuka Y, Yamatogi Y, Oka E. The early infantile epileptic encephalopathy with suppression-burst: developmental aspects. Brain Dev 1987;9:371-6.

10 Aicardi J. Early myoclonic epilepsy. In: Rojer J, Dravet C, Bureau M, Dreifuss FE, Wolf $\mathrm{P}$, eds. Epileptic syndromes in infancy, childhood and adolescence. London: Libbey, 1985: 12-21.

11 Young ID, McKeever PA, Squier MV, Grant J. Lethal olivopontoneocerebellar hypoplasia with dysmorphic features in sibs. $\mathcal{F}$ Med Genet 1992;29:733-5.

12 Barth PG, Vrensen GFJM, Uylings HBM,Oorthuys JWE, Stam FC. Inherited syndrome of microcephaly, dyskinesia and pontocerebellar hypoplasia: a systemic atrophy with early onset. F Neurol Sci 1990;104:25-42. 